\title{
Organisation spatiale de la diversité génétique et flux polliniques dans une chênaie mixte
}

\author{
Réjane Streiff, Alexis Ducousso, Antoine Kremer*
}

Institut national de la recherche agronomique, laboratoire de génétique et d'amélioration des arbres forestiers. Pierroton, 33610 Cestas, France

\begin{abstract}
Spatial genetic structure and pollen gene flow in a mixed oak stand. The spatial genetic structure was analysed in a 6 ha mixed stand of 355 adult oaks trees (Quercus petraea (Matt.) Liebl. and Quercus robur L.). The genetic diversity was investigated with six highly polymorphic microsatellite loci (15 to 32 alleles per locus). Two parameters of the spatial genetic structure were used: the genetic differentiation $(F s t)$, for different spatial scales within the stand, and the spatial autocorrelation $(I)$ between the genotypes in the stand. Both species are characterised by a low but significant genetic differentiation, whatever the spatial scale of observation (except for the smallest scale of $<10 \mathrm{~m}$ for Quercus robur). The autocorrelation values are significant for the smallest scales for Quercus petraea and the spatial structure appears weaker for Quercus robur. Results are interpreted in terms of gene flow for both species. Paternity analysis based on multilocus microsatellite data made it possible to identify the paternal parents of progenies collected on 13 mother trees in the stand. Results of the paternity analysis indicate that pollen flow combines dispersion at short distances and transport at long distances. As a result, the spatial structure observed is seen as the consequence of the asymmetry of pollen versus seed flow. Restricted seed movement is likely to build up spatial structures that are disrupted by long distance pollen movements. (C) Inra/Elsevier, Paris
\end{abstract}

genetic differentiation / spatial autocorrelation / paternity analysis / pollen flow / Quercus petraea / Quercus robur

Résumé - L'organisation de la diversité génétique a été décrite dans le cas d'un peuplement mélangé de chêne sessile et pédonculé constitué de 355 arbres adultes répartis sur une surface de 6 ha. La diversité a été appréhendée à l'aide de 6 loci microsatellites manifestant un polymorphisme très élevé (de 15 à 32 allèles par locus). L'organisation a été décrite à l'aide de deux paramètres : la différenciation $(F s t)$ entre différentes subdivisions spatiales de la parcelle étudiée et l'autocorrélation spatiale $(I)$ entre les génotypes présents sur la parcelle. Les deux espèces se caractérisent

* Correspondance et tirés à part : email : Antoine.Kremer@inra.pierroton.inra.fr, Tél. 0557979074 , fax 0557979088 
par une différenciation génétique faible mais significative, quelle que soit la taille des subdivisions spatiales considérées (à l'exception des faibles tailles $<10 \mathrm{~m}$ chez le chêne pédonculé). L'autocorrélation est significative aux faibles distances chez le chêne sessile alors qu'une structuration plus faible apparaît chez le chêne pédonculé. Ces valeurs sont interprétées en fonction des flux de gènes chez les deux espèces, dont des indications sont données pour les flux de pollen. En effet des recherches de paternité à l'aide des données multilocus sur les microsatellites ont permis d'identifier les parents pollinisateurs de treize descendances récoltées dans la parcelle. Ces résultats suggèrent que les flux de pollen cumulent une dispersion à courte distance et des transports à longue distance. La faible structuration spatiale est interprétée comme le résultat de l'asymétrie de flux entre graines et pollen. La structure mise en place par la dispersion réduite des graines est érodée par les flux de pollen à longue distance. (C) Inra/Elsevier, Paris

différenciation génétique / autocorrélation spatiale / recherche de paternité / flux de pollen / microsatellites / Quercus petraea / Quercus robur

\section{INTRODUCTION}

La régénération par ensemencement naturel est la pratique sylvicole utilisée pour renouveler les chênaies, quand la production régulière et homogène des graines est suffisante (Lanier, 1994). Les conséquences génétiques de cette pratique sur l'évolution de la diversité sont très largement méconnues. On peut en particulier s'interroger sur l'effet cumulatif des régénérations naturelles sur l'introduction d'une consanguinité dans le peuplement forestier concerné, souvent décrite par les forestiers par le terme de «cercles de consanguinité». En effet, en cas de dispersion limitée du pollen et/ou des graines, on conçoit aisément que des relations d'apparentement soient maintenues et renforcées au fil des régénérations. Or la dépression de consanguinité est particulièrement sévère pour des caractères liés à la valeur adaptative chez les arbres forestiers (Durel et al., 1996). Cette préoccupation très appliquée relève en fait d'une certaine complexité dans son approche expérimentale, dès lors que les générations des espèces concernées sont extrêmement longues. Il est illusoire de vouloir suivre les filiations sur plus d'une génération et de pouvoir estimer les niveaux de consanguinité. En revanche si les apparentements entre arbres voisins se renforcent au cours des générations, alors une organisation spatiale de la diversité génétique devrait apparaître au sein des chênaies. La mise en place d'une structuration spatiale résulte essentiellement de l'interaction entre flux de gènes, pressions de sélection locale et dépression de consanguinité. Dans le cas des chênes, les flux de gènes et surtout leur asymétrie (Ennos, 1994) peuvent agir dans des directions opposées vis à vis de la structuration spatiale. D'une part les flux restreints de graines conduiront inévitablement à l'instauration d'une structuration spatiale, alors que les flux de pollen à plus large distance tendront à rompre de telles structures. Nous nous proposons dans cette contribution de mettre en perspective l'organisation spatiale de la diversité intrapopulationnelle avec les flux de pollen chez deux espèces majeures de la forêt européenne : le chêne sessile (Quercus petraea (Matt.) Liebl.) et le chêne pédonculé (Quercus robur L.).

Plusieurs études menées sur d'autres espèces forestières soulignent la quasi absence de structuration spatiale de la diversité au stade adulte du peuplement (Merzeau et al., 1994, Leonardi et Menozzi, 1996 chez le hêtre; Berg et 
Hamrick, 1995 chez un chêne américain). Les raisons généralement invoquées sont les pressions de sélection tendant à éliminer les arbres consanguins, et les flux de pollen. Dans notre étude relative aux deux chênes nous proposons d'étudier les flux de pollen par recherche de paternité à partir de marqueurs microsatellites (Dow et al., 1995). Cette méthode permet d'estimer les flux efficaces contrairement aux anciennes méthodes se limitant à la dispersion purement physique du pollen (Campbell, 1991). Par ailleurs elle se place au niveau de la plante receveuse de pollen (approche puits) et comptabilise donc tous les événements de fécondation y compris ceux résultant de pollinisation à longue distance.

\section{MATÉRIEL ET MÉTHODES}

\subsection{Peuplement expérimental}

La description de l'organisation spatiale porte sur un peuplement mixte constitué de chêne sessile et de chêne pédonculé, comprenant au total 355 arbres d'âge supérieur à 120 ans. Le peuplement expérimental est de forme carrée et couvre une surface de 5,76 ha. Il fait partie de la forêt de la Petite Charnie (département de la Sarthe) et est entouré sur deux de ses côtés d'autres parcelles de chênes - des deux espèces - en âge de fleurir. L'échantillonnage du peuplement expérimental est exhaustif, et les coordonnées de chaque arbre ont été relevées (figure 1).

L'analyse des caractères de morphologie foliaire a permis d'identifier au plan taxinomique chaque arbre (Bacilieri et al., 1995) à l'exception de six d'entre eux dont le statut taxinomique n'a pu être défini. Ces derniers manifestaient en effet des caractères morphologiques intermédiaires entre les deux espèces. L'effectif se décompose de la manière suivante : 166 chênes pédonculés, 183 chênes sessiles.

Sur treize arbres adultes distribués sur l'ensemble de la parcelle, une récolte de graines a été effectuée à l'automne 1994 (figure 1). Ces graines ont été semées en pépinière et les extractions d'ADN ont été faites sur des semis de deux ans (une centaine au maximum par descendance) pour les recherches de paternité. Entre les deux analyses (structuration spatiale et paternité), une éclaircie avait été réalisée dans le peuplement prélevant 59 arbres. Les analyses de paternité ont donc porté sur un peuplement adulte composé de 296 arbres (124 petraea, 167 robur et 5 intermédiaires).

\subsection{Analyses moléculaires}

Des feuilles de chaque arbre et de chaque semis ont été récoltées et stockées à $-80^{\circ} \mathrm{C}$. L'ADN a été extrait en utilisant la méthode de Doyle and Doyle (1987), simplifiée par Bodénès et al. (1996). Les réactions d'amplification ont été réalisées dans un volume de $25 \mu \mathrm{L}$, comprenant $0,2 \mu \mathrm{M}$ de chaque amorce, $67 \mathrm{mM}$. de Tris $\mathrm{HCl}, \mathrm{pH} 8,1,2 \mathrm{mM}$ de $\mathrm{MgCl}_{2}, 1 \mathrm{ng}$ de BSA, 0,2 \% de $\beta$-mercaptoéthanol, $16 \mathrm{mM}$ d'acétate d'ammonium, $100 \mu \mathrm{M}$ de chaque dNTP, et 0,8 unités de Taq polymérase (Gibco BRL). Le protocole d'amplification comprend une phase de dénaturation à $94^{\circ} \mathrm{C}$ durant $4 \mathrm{~min}$, suivie par 30 cycles, comprenant chacun une étape de dénaturation $\left(94^{\circ} \mathrm{C}\right.$ pendant $\left.45 \mathrm{~s}\right)$, une étape 


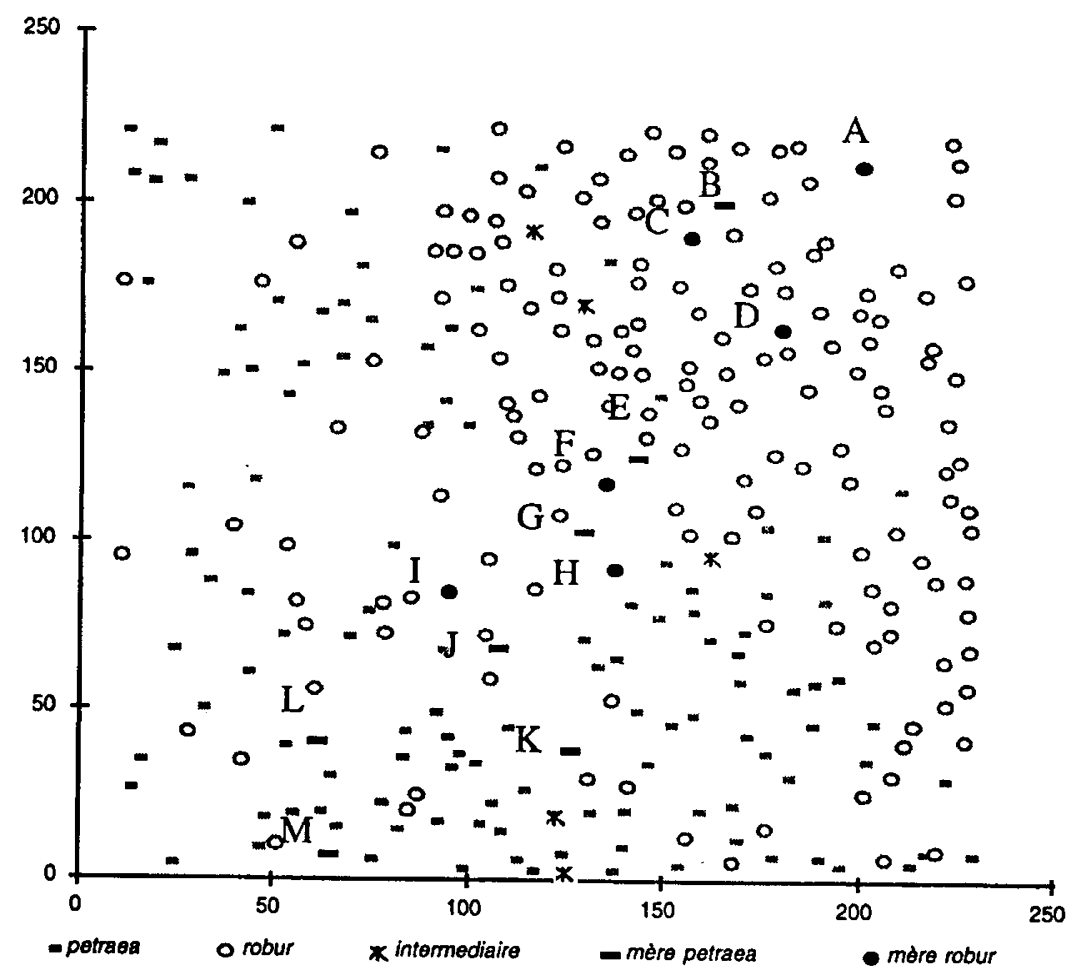

Figure 1. Distribution spatiale des arbres adultes du peuplement expérimental. $\circ$ : Chêne pédonculé; : Chêne sessile; $\bullet$ : Chêne pédonculé dont des descendances ont été récoltées; $:$ : Chêne sessile dont des descendances ont été récoltées.

d'hybridation $\left(50{ }^{\circ} \mathrm{C}\right.$ pendant $45 \mathrm{~s}, 55^{\circ} \mathrm{C}$ pour le locus $\left.\mathrm{AG} 1 / 5\right)$ et une étape d'élongation $\left(72^{\circ} \mathrm{C}\right.$ pendant $\left.30 \mathrm{~s}\right)$.

Les produits d'amplification sont alors dénaturés à $94^{\circ} \mathrm{C}$ pendant 4 min dans une solution contenant $95 \%$ de formamide, $0,05 \%$ de bleu de Bromophénol, $0,05 \%$ de xylène cyanol et $10 \mu \mathrm{M}$ de soude. Les électrophorèses sont conduites sur des gels à $6 \%$ de polyacrylamide en conditions dénaturantes (urée $6 \mathrm{M}$ ) colorés au nitrate d'argent (Bodénès et al., 1996).

\subsection{Marqueurs microsatellites}

Les analyses ont porté sur six locus microsatellites, quatre issus des travaux de Steinkellner et al. (1997) sur le chêne sessile (locus AG104, AG9, AG1/5, AG36) et deux autres issus des travaux de Dow et al. (1995) sur un chêne blanc américain (Quercus macrocarpa) (locus MSQ4 et MSQ13). Tous ces locus comprennent des répétitions de type (AG)n. Tous les arbres adultes (296) et les descendants élevés en pépinière (984 récoltés sur 13 familles) ont été typés pour ces six locus. 


\subsection{Analyse de l'organisation spatiale de la diversité}

Le terme d'organisation spatiale de la diversité sous entend deux notions complémentaires : celle de différenciation et celle de structuration spatiale. La première ne rend compte que du niveau de l'hétérogénéité entre les différentes unités spatiales, alors que la seconde traduit l'organisation de cette hétérogénéité entre les différentes unités. Plus précisément, la structuration se fixe pour objectif de comparer la similarité génétique entre unités et leur distance physique. Nous avons utilisé comme mesure de la différenciation le paramètre $F s t$ de Wright (1969) et comme mesure de la structuration l'autocorrelation spatiale (Indice de Moran I, Sokal et Oden, 1978).

\subsubsection{Différenciation spatiale}

Le paramètre de différenciation Fst (Weir et Cockerham, 1984) a été calculé à différentes échelles spatiales : le peuplement a été artificiellement subdivisé en carrés de $K \mathrm{~m}$ de coté. Les valeurs de $K$ varient de 20 à $120 \mathrm{~m}$ par pas de $10 \mathrm{~m}$. À chaque valeur de $K$, la différenciation $F s t$ entre dèmes (un dème correspond à la population constituée par les arbres situés à l'intérieur d'un carré) est estimée selon la méthode de Weir et Cockerham (1984), basée sur une analyse de variance. Pour chaque allèle, la variabilité observée est décomposée selon le modèle hiérarchique à effets aléatoires suivant

$$
Y_{i j k}=\mu+d_{i}+z_{i j}+g_{i j k}
$$

où $d_{i}, z_{i j}, g_{i j k}$ représentent respectivement les effets dèmes, zygote (intradème) et allèle (intradème et intrazygote). Fst est alors défini, à cet allèle, de la manière suivante :

$$
F s t=\frac{\sigma_{d}^{2}}{\sigma_{d}^{2}+\sigma_{z}^{2}+\sigma_{g}^{2}}
$$

L'estimation des valeurs de $F s t$ a été faite séparément au niveau de chaque locus et de chaque allèle. Une estimation globale multilocus et multiallélique de $F$ st est faite de la manière suivante (Weir et Cockerham, 1984)

$$
\widehat{F s t}=\frac{\Sigma \widehat{\sigma}_{d}^{2}}{\Sigma \widehat{\sigma}_{d}^{2}+\widehat{\sigma}_{z}^{2}+\widehat{\sigma}_{g}^{2}}
$$

où $\Sigma$ indique la somme sur tous les allèles de tous les locus.

Parmi les différents estimateurs possibles, ce dernier proposé par Weir et Cockerham (1984) s'avère être le moins biaisé (Goudet et al., 1996).

\subsubsection{Structuration spatiale}

L'estimation de la différenciation entre les différents dèmes ne permet pas de préciser si les différences entre dèmes sont spatialement organisées. Nous avons utilisé pour cela l'autocorrélation spatiale (ou indice de Moran $I$ ) de Sokal et 
Oden (1978). La définition de $I$ pour un allèle donné, et pour des arbres espacés d'une distance de $k \mathrm{~m}$, est la suivante :

où $W=\sum_{i=1}^{n} \sum_{j \neq i}^{n} w_{i j}$

$$
I_{k}=\frac{n \sum_{i=1}^{n} \sum_{j \neq i}^{n} w_{i j}\left(x_{i}-\bar{x}\right)\left(x_{j}-\bar{x}\right)}{W \sum_{i=1}^{n}\left(x_{i}-\bar{x}\right)^{2}}
$$

$n$ est le nombre total d'arbres, et $w_{i j}$ est égal à 1 si les arbres $i$ et $j$ sont séparés par une distance variant de $k+10 \mathrm{~m}$ à $k-10 \mathrm{~m}$, et à 0 dans tous les autres cas. $x_{i}$ et $\bar{x}$ représentent la valeur de l'arbre pour l'allèle concerné et la moyenne de l'ensemble des arbres. Chaque arbre peut prendre trois valeurs possibles ( 1 s'il est homozygote à l'allèle concerné, 0,5 s'il est hétérozygote à l'allèle concerné, et 0 s'il ne possède pas cet allèle au locus considéré).

L'estimateur multilocus et multiallélique est obtenu en faisant le rapport de la somme des covariances sur la somme de toutes les variances. L'indice de Moran $I$, qui correspond à ce coefficient d'autocorrélation spatiale est calculé séparément pour différentes classes de distance.

\subsubsection{Tests statistiques de la différenciation et de la structuration spatiale}

Un test statistique empirique a été construit pour tester l'hypothèse nulle d'absence de différenciation ou de structuration. Pour construire l'hypothèse nulle, les génotypes multilocus sont distribués au hasard sur les arbres dont les emplacements sont maintenus : les données génétiques sont permutées sur les données de localisation physique (coordonnées). Ces permutations sont réalisées 1000 fois. À chaque permutation, les valeurs de Fst et de $I$ sont calculées selon les méthodes de Weir et Cockerham (1984) et de Sokal et Oden (1978). La distribution des 1000 valeurs obtenues constitue la distribution empirique de l'hypothèse nulle, à laquelle peuvent être comparées les valeurs observées de Fst et de $I$.

\subsection{Recherche de paternité}

La recherche de paternité se fait par comparaison des haplotypes multilocus d'origine mâle de chaque descendant avec tous les haplotypes possibles des 296 parents potentiels. L'haplotype d'origine mâle est obtenu par soustraction de l'haplotype d'origine femelle (connu, correspondant à l'arbre sur lequel la descendance a été récoltée) du génotype du descendant. Les comparaisons permettent ainsi d'exclure les parents potentiels les uns après les autres, aboutissant généralement à l'identification d'un seul parent n'ayant pu être exclu. Sur les 984 descendants analysés, 17 furent affectés à plus d'un seul père par la procédure d'exclusion. Ces 17 semis ont été soustraits à l'analyse par la suite. La recherche de paternité étant faite à l'aide de 6 loci très variables (plus de 15 allèles par locus), l'assignation de parenté est très précise. D'une part, 
la probabilité d'exclure un parent mâle si ce n'est pas le père est de 0,99968 (Streiff et al., 1998a). D'autre part, la probabilité pour qu'un père détecté à l'intérieur de la parcelle ne soit pas le vrai père (le vrai étant à l'extérieur de la parcelle étudiée) est dans plus de $80 \%$ des semis inférieure à $5 \%$.

\section{RÉSULTATS}

Les six locus étudiés sont extrêmement polymorphes. Dans la cohorte formée des 355 arbres adultes, de 15 à 32 allèles ont été identifiés et les valeurs d'hétérozygotie théorique varient entre $70 \%$ et $87 \%$. Les allèles sont partagés par les deux espèces, à l'exception de certains allèles rares. Ce faible niveau de différenciation génétique entre espèces confirme les résultats déjà obtenus sur ce peuplement (Bacilieri et al., 1994) et sur d'autres peuplements de l'aire naturelle (Zanetto et al., 1994) à l'aide des isozymes.

\subsection{Différenciation spatiale}

Les valeurs moyennes de $F s t$ sont significativement différentes de 0 quelle que soit la distance considérée chez le chêne sessile (figure 2). On note toutefois des valeurs plus élevées aux faibles classes de distance (inférieures à $70 \mathrm{~m}$ ). Le pattern suivi par le chêne pédonculé est sensiblement différent. Les valeurs observées de $F$ st ne sont pas significativement différentes de zéro aux très faibles distances, alors qu'elles s'écartent d'une différenciation nulle aux distances plus élevées.

Bien que la variabilité des Fst entre locus soit plus élevée aux faibles distances, due essentiellement à des tailles d'échantillonnage plus faibles par dème, l'évolution des courbes de Fst ne manifeste pas de grande hétérogénéité entre locus (figure 3).

\subsection{Structuration spatiale}

La différenciation spatiale estimée à l'aide des $F$ statistiques est spatialement organisée, comme le suggère la comparaison des valeurs de $F s t$ et des indices de Moran $I$. On remarque ainsi une similarité génétique plus forte aux faibles distances chez le chêne sessile, qui est beaucoup plus atténuée chez le chêne pédonculé (figure 4). L'hétérogénéité entre locus est plus forte pour les valeurs de $I$ que pour celles de Fst (figure 5). La structuration observée chez le chêne sessile sur l'ensemble des locus (figure 4) est en partie due à celle très élevée pour le locus MSQ4 (figure 5). Comme attendu par la théorie (Cliff et Ord, 1981) la valeur espérée de l'indice de Moran sous l'hypothèse nulle est négative $(-1 /(n-1))$ où $n$ est le nombre d'arbres considérés pour le calcul de $I$.

Les statistiques de différenciation (2) et de structuration (4) ont été comparées à celles prenant en compte le fait que les allèles peuvent dériver les uns des autres par ajout ou suppression d'une unité de répétition (stepwise mutation model, Valdes et al., 1993) dans le motif microsatellite. Ces nouvelles statistiques sont Rst (Slatkin, 1995) pour la différenciation et $I q$ pour l'autocorrélation ( $I q$ est l'indice de Moran calculé sur la taille du fragment). Les comparaisons entre les différentes statistiques sont présentées dans un article 

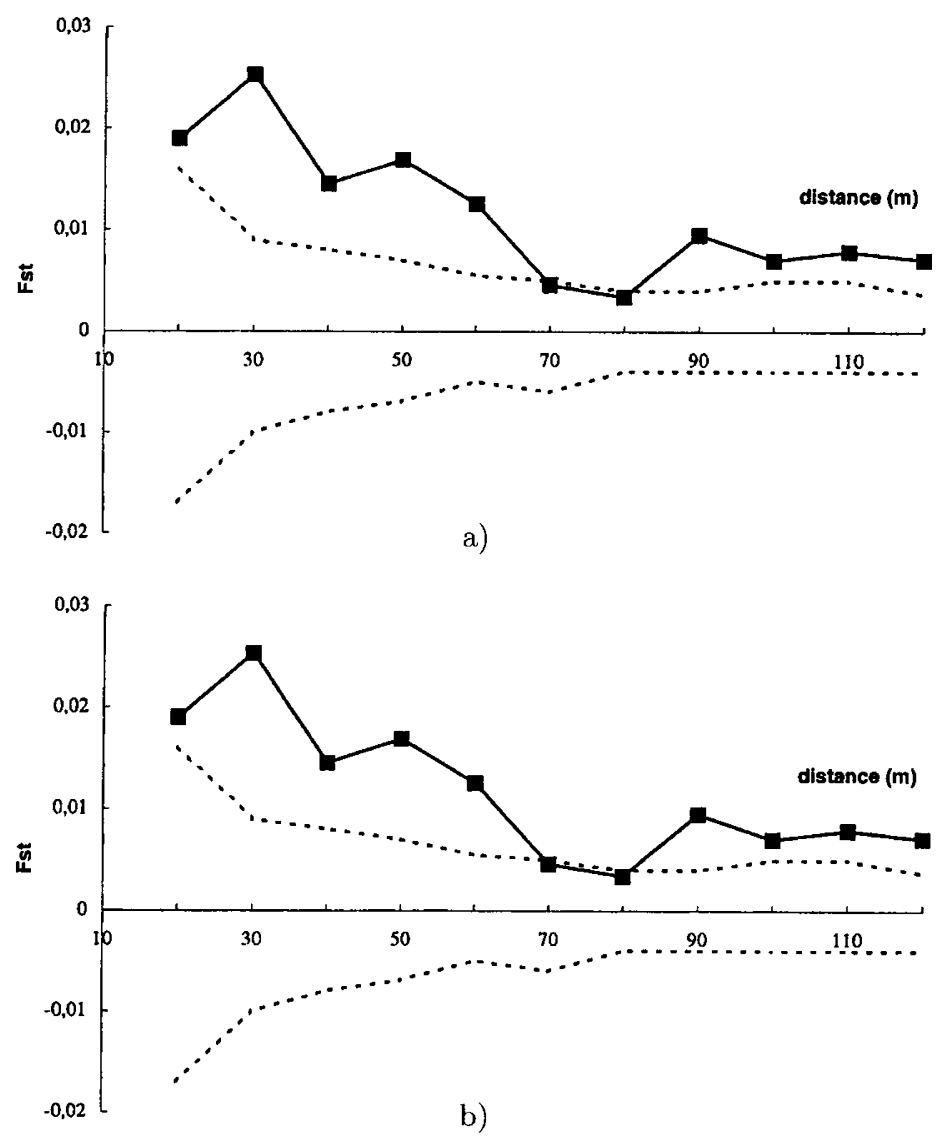

Figure 2. Évolution des valeurs moyennes de Fst avec la taille de la maille (distance sur le graphique) de la subdivision. Les deux courbes en tirets délimitent l'enveloppe formée par $95 \%$ des valeurs de $F s t$ obtenues par les 1000 permutations (voir texte). a) Chêne sessile; b) Chêne pédonculé.

consacré à ce sujet (Streiff et al., 1998b). En résumé, les variations de Rst et de $I q$ en fonction des distances sont parallèles à celle de Fst et de $I$. Par ailleurs, les variances d'échantillonnage associées à $R s t$ et à $I q$ sont beaucoup plus élevées que celles associées à $F s t$ et $I$. C'est la raison pour laquelle nous avons choisi de ne présenter que les variations de $F$ st et de $I$.

\subsection{Flux polliniques (tableau I et figure 6)}

Dans le cas du chêne pédonculé, pour lequel 447 descendants ont été récoltés sur six arbres mères, les recherches de paternité ont permis d'attribuer un père à 152 descendants (34 \%). Pour tous les autres, les parents mâles sont situés en dehors de la parcelle. Dans le cas du chêne sessile, les pères de 158 descendants 


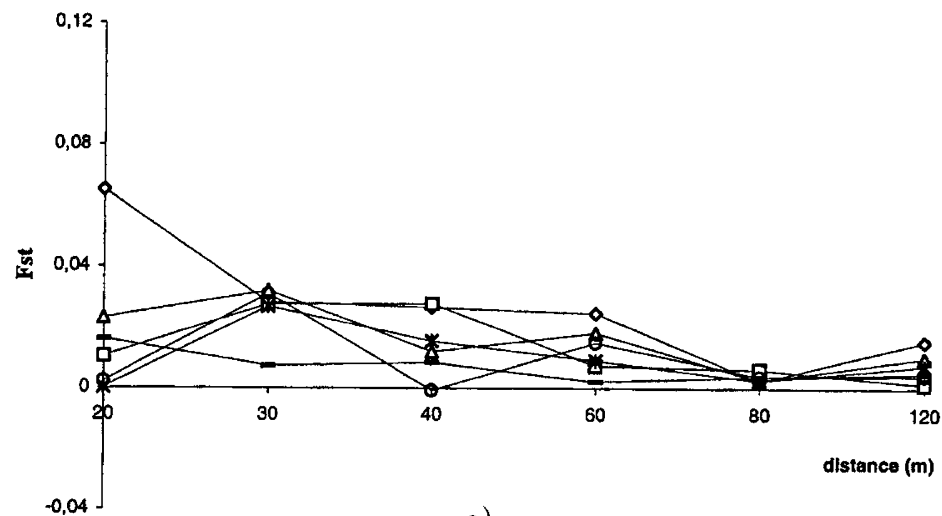

a)

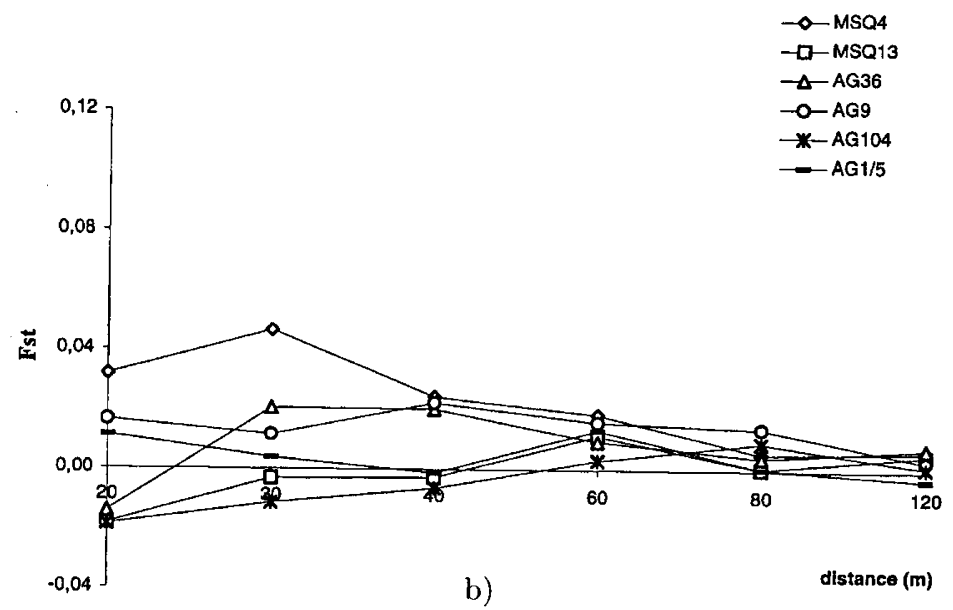

Figure 3. Évolution des valeurs de $F s t$ relatives à chaque locus avec la taille de la maille (distance sur le graphique) de la subdivision. a) Chêne sessile; b) Chêne pédonculé.

sur 537 (soit $29 \%$ ) ont été retrouvés parmi les 296 arbres adultes de la parcelle. Une étude plus détaillée de la répartition spatiale des pères fait apparaître une localisation préférentielle dans le voisinage immédiat de l'arbre mère (Streiff et al., soumis). Bien que significative au plan statistique par rapport à l'hypothèse nulle de distribution aléatoire des pères, la tendance à la proximité des pères autour des arbres mères reste très timide. Par ailleurs, une très forte hétérogénéité peut être observée entre les différentes mères considérées : pour certaines d'entre elles, les arbres pollinisateurs sont relativement proches, alors que pour d'autres ils sont distribués de manière très diffuse. Cette hétérogénéité est illustrée par les deux exemples de la figure 6 . Ainsi dans le cas de l'arbre C, on remarquera une dispersion des arbres pollinisateurs montrant deux pics : un premier ensemble principalement constitué de pères identifiés dans la parcelle et relativement proches, et le reste provenant de l'extérieur de la parcelle. Les grains de pollen pollinisant le second arbre $(G)$ proviennent d'arbres situés à 

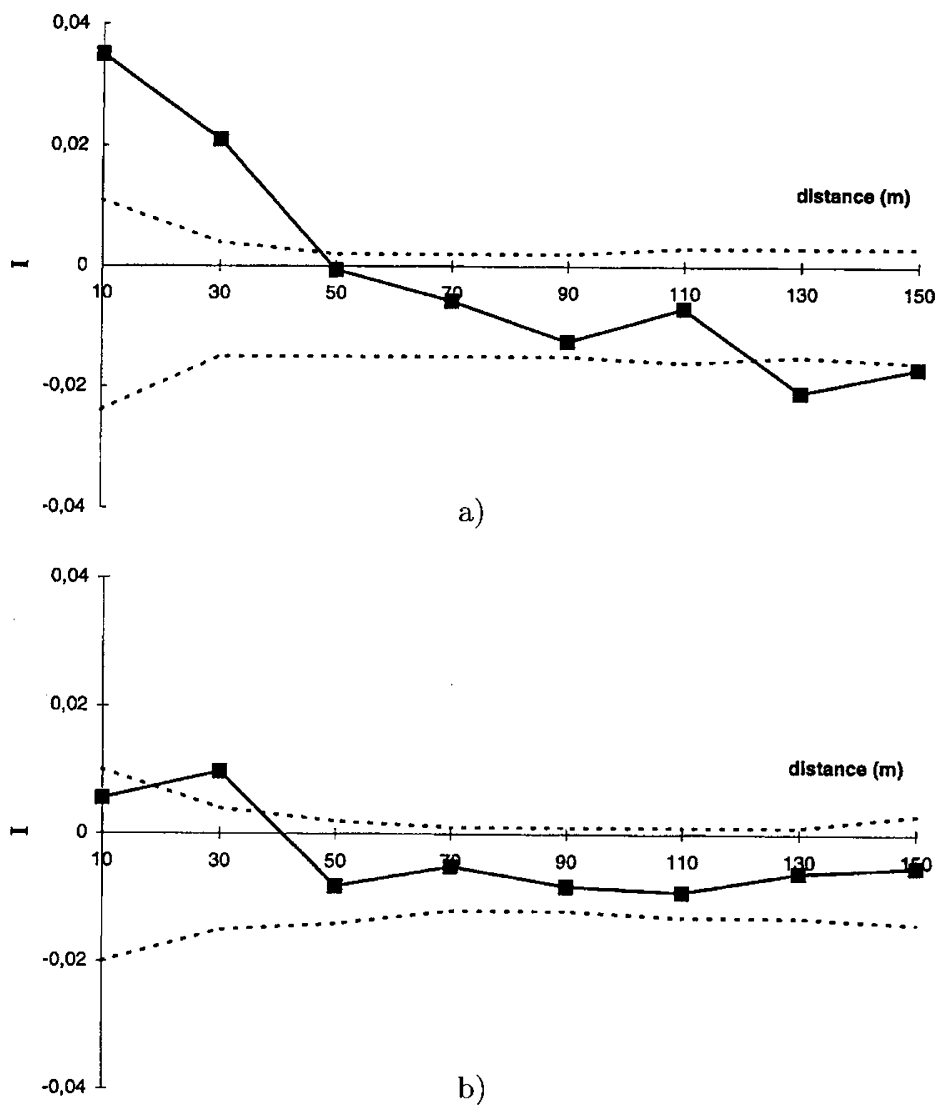

Figure 4. Évolution des valeurs moyennes de l'indice de Moran $(I)$ avec la classe de distance séparant les arbres. Les deux courbes en tirets délimitent l'enveloppe formée par $95 \%$ des valeurs de Fst obtenues par les 1000 permutations (voir texte). a) Chêne sessile b) Chêne pédonculé

des distances variables. L'effet d'une proximité des arbres pollinisateurs est nettement moins marqué pour cet arbre.

\section{DISCUSSION ET CONCLUSION}

L'analyse simultanée de la différenciation et de la structuration spatiale chez les deux chênes, sessile et pédonculé, s'est soldée par deux résultats principaux. D'une part les deux espèces manifestent une différenciation faible mais significative à toutes les échelles spatiales d'analyse, d'autre part cette différenciation est spatialement structurée aux faibles distances et surtout dans le cas du chêne sessile. Même si les analyses de différenciation et de structuration ont été rarement conduites de manière simultanée, les conclusions d'étude particulière sur l'un ou l'autre aspect de l'organisation spatiale de la diversité, relatifs à d'autres arbres forestiers, sont similaires aux nôtres 

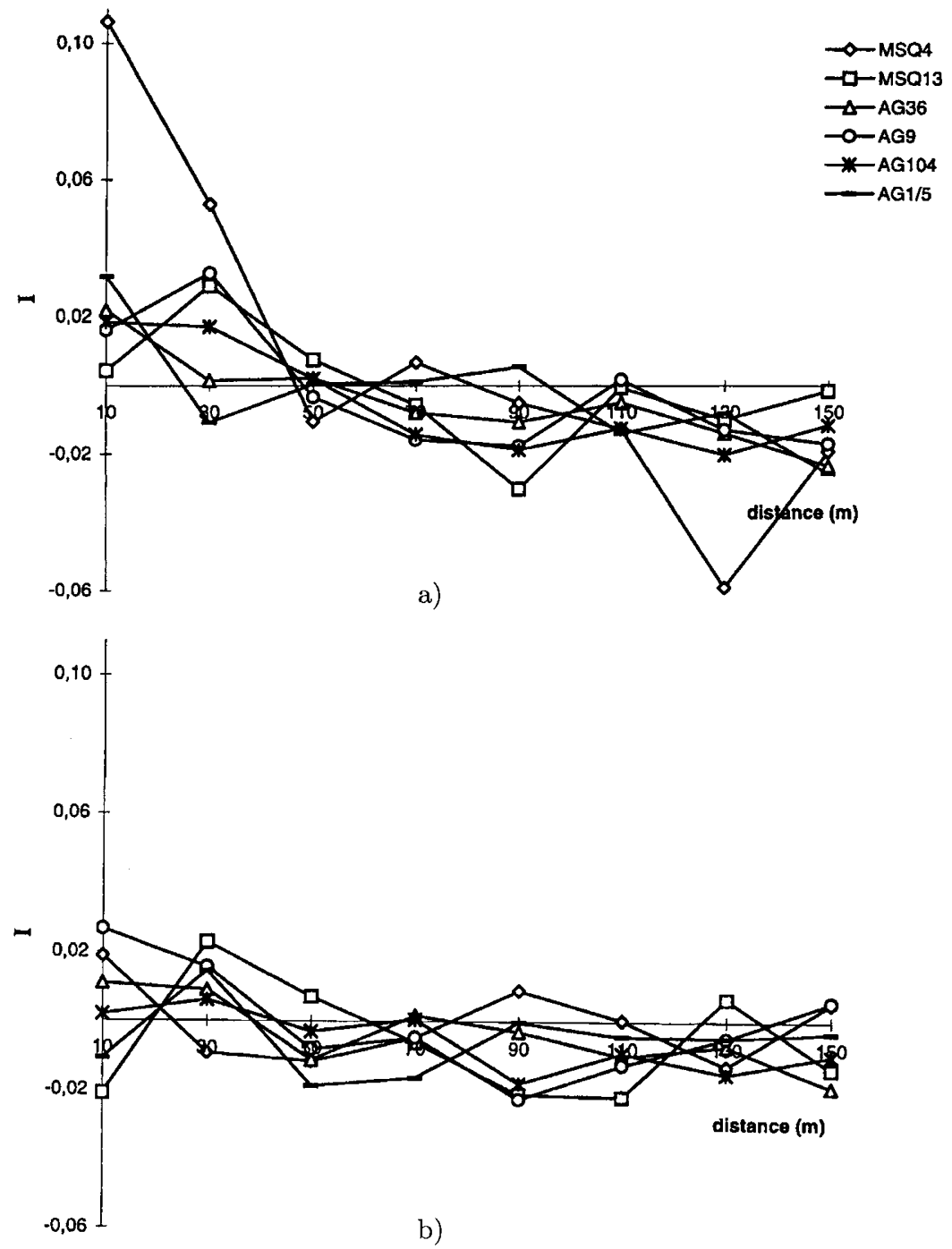

Figure 5. Évolution des valeurs de l'indice de Moran $(I)$ relatives à chaque locus avec la classe de distance séparant les arbres. a) Chêne sessile; b) Chêne pédonculé.

(Schnabel et al., 1991 sur Gleditsia triachantos, Merzeau et al., 1994, Leonardi et Menozzi, 1996 sur le hêtre, Berg et Hamrick, 1995 sur Quercus leavis). Nos résultats obtenus à l'aide de marqueurs microsatellites étaient déjà suggérés par ceux issus d'une étude portant sur les allozymes dans le même peuplement (Bacilieri et al., 1994; Streiff et al., 1998b). La concordance entre observations faites avec des marqueurs différents, en dépit d'une certaine hétérogénéité entre loci, impose l'hypothèse d'effets systématiques indépendants des zones génomiques considérées. Ce caractère systématique exclut les effets de sélection microenvironnementale, propres à la variation des conditions pédologiques dans 
Table I. Résultats de l'analyse de paternité

\begin{tabular}{lcccc}
\hline Espèce & Arbre mère & N & N1 & Nob \\
\hline Q. robur & & & & \\
& A & 52 & 14 & 8 \\
& C & 93 & 47 & 16 \\
D & 55 & 21 & 16 \\
Q. petraea & F & 75 & 21 & 14 \\
& H & 91 & 25 & 14 \\
& I & 81 & 24 & 9 \\
& & & & \\
& B & 91 & 21 & 15 \\
& E & 96 & 20 & 13 \\
& G & 91 & 32 & 23 \\
& I & 91 & 23 & 15 \\
& K & 92 & 35 & 13 \\
& L & 28 & 18 & 4 \\
& M & 48 & 9 & 4 \\
\hline
\end{tabular}

$\mathrm{N}$ : nombre de descendants analysés ; N1 : nombre de descendants dont le père identifié par recherche de paternité se trouve dans la parcelle d'étude; Nob : nombre de pères ayant participé à la pollinisation des $\mathrm{N} 1$ descendants.

la parcelle considérée, comme cause potentielle de la différenciation et de la structuration. Il faut donc invoquer soit le régime de reproduction (autogamie potentielle), soit les flux géniques pour interpréter nos résultats. Ces deux espèces de chênes étant strictement allogames (Bacilieri et al., 1995), les flux de gènes restent la seule alternative possible.

Le maintien d'une différenciation significative mais réduite aux faibles distances chez les deux espèces pourrait résulter des flux très asymétriques entre pollen et graines. Les données expérimentales disponibles sur les niveaux comparés de différenciation ( $G s t$ ) entre génome nucléaire et génome cytoplasmique à l'échelle de l'aire de distribution sont en effet très contrastées. Les valeurs obtenues chez le chêne sessile et pédonculé varient pour les isozymes entre 1,7\% et $3,2 \%$ selon les échantillons de populations analysées (Kremer et al., 1991; Zanetto et al., 1994; Zanetto et Kremer, 1995; Le Corre et al., 1997). À l'inverse, les valeurs de différenciation pour les génomes chloroplastiques se situent entre $83 \%$ et $89 \%$ (Kremer et al., 1991; Petit et al., 1993; Dumolin-Lapègue et al., 1997). En supposant que les populations soient à l'équilibre entre mutation dérive et migration ces valeurs peuvent être interprétées et comparées en termes de taux de migration. C'est ce qui a été réalisé par Ennos (1994) et El Mousadik et Petit (1996) qui obtiennent des rapports de taux de migration pollen/graines de l'ordre de 200 à 500 ! Nulle autre plante citée par ces auteurs ne manifeste un tel niveau d'asymétrie entre flux de pollen et de graines. Par ailleurs l'asymétrie est plus élevée chez le chêne sessile que chez le chêne pédonculé (El Mousadik et Petit, 1996). Ces taux relatifs de migration estimées a posteriori à partir du modèle en îles de Wright (1969), méritent cependant d'être confrontés aux données a priori obtenues par observation directe des flux. Nos propres résultats, présentés dans cette contribution, suggèrent que les flux de pollen sont très importants et très hétérogènes. Plus de $60 \%$ du pollen provenait, en moyenne, de l'extérieur de la parcelle d'étude d'une 


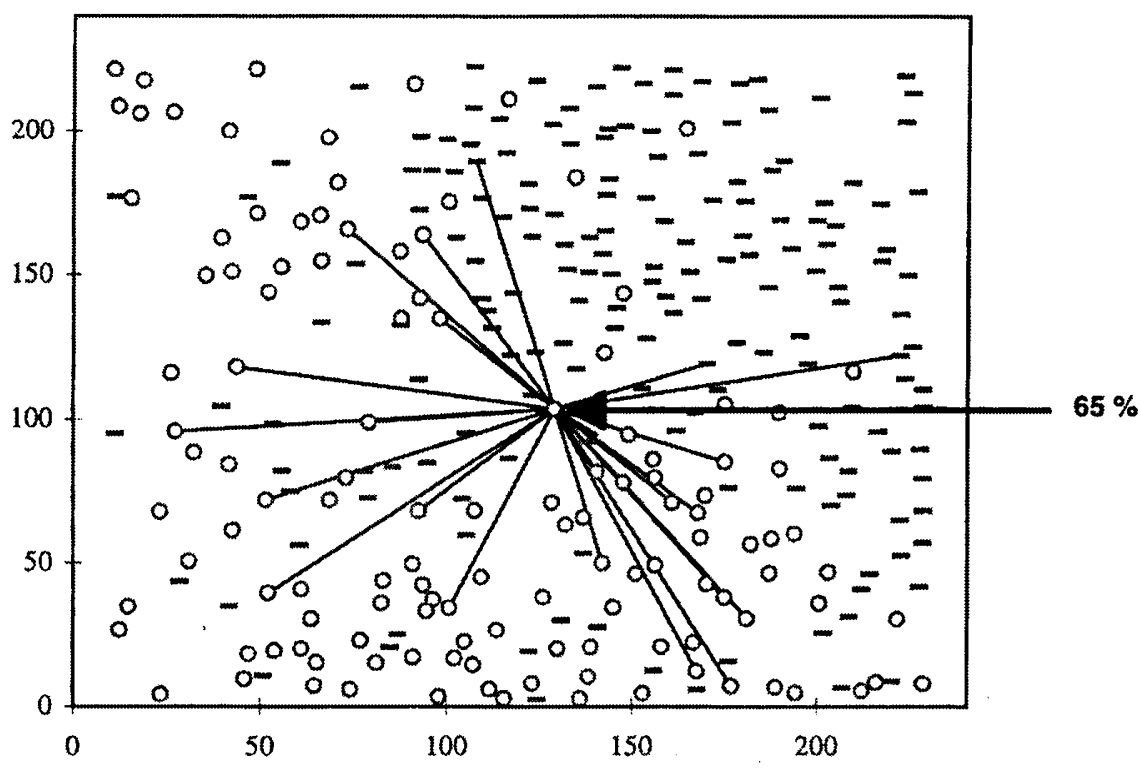

a)

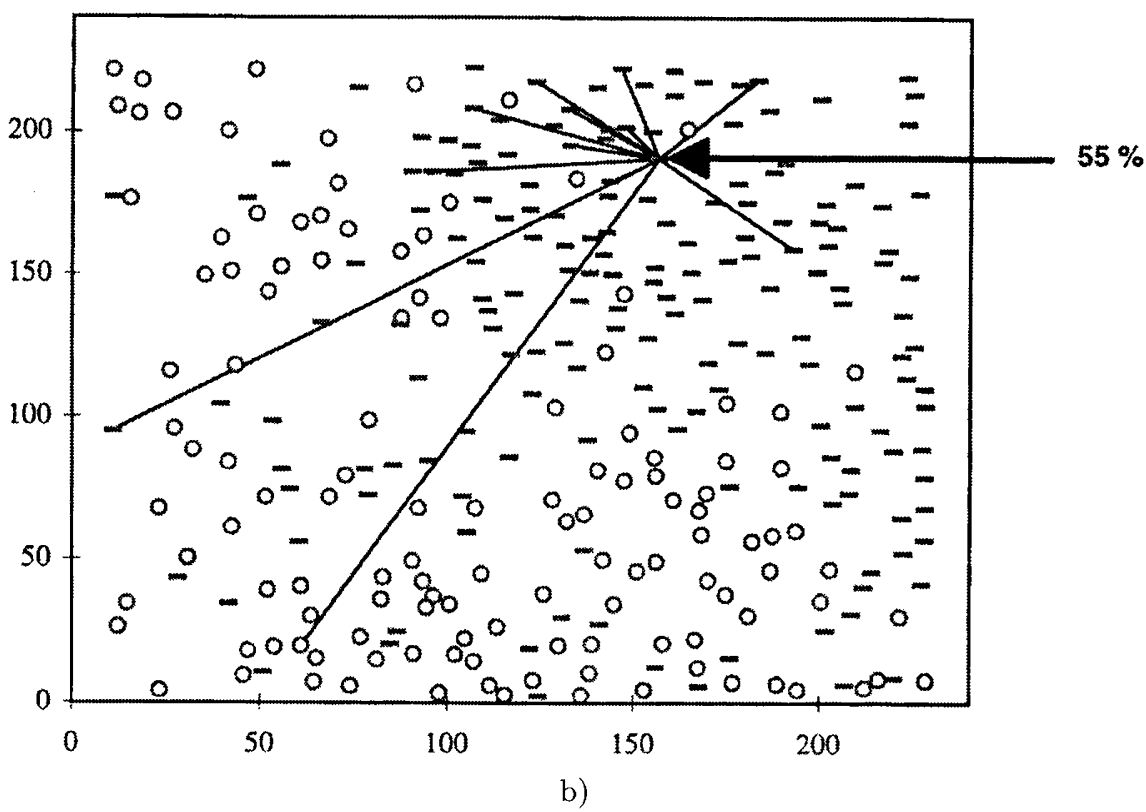

Figure 6. Répartition spatiale des arbres pollinisateurs de deux descendances. Les traits relient l'arbre pollinisateur avec l'arbre mère sur lequel la descendance a été récoltée (voir figure 1 également). La flèche matérialise le pollen provenant de l'extérieur de la parcelle et les pourcentages, la proportion de pollen venant de l'extérieur : a) descendance de l'arbre G (sessile) ; b) descendance de l'arbre C (pédonculé). 
surface de 6 ha et l'hypothèse de dispersion à longue distance ne peut être exclue. Les flux de pollen cumuleraient donc une dispersion à courte distance (croisements préférentiels entre arbres voisins) et une dispersion à longue distance. Ces derniers constituent indéniablement une force tendant à détruire toute structure spatiale. Malheureusement nous ne disposons pas des données biologiques relatives à la dissémination des glands. Des recherches de maternité viennent cependant d'être réalisées récemment chez un chêne américain (Quercus macrocarpa, Dow et Ashley, 1996) et confirment la dispersion nettement plus réduite des graines par rapport au pollen : $82 \%$ des semis en forêt avaient leur mères à moins de $30 \mathrm{~m}$. On pourrait donc en conclure que les flux réduits de graines tendent à instaurer une structure spatiale, qui est progressivement érodée par les flux de pollen. Les relations entre structure spatiale observée et asymétrie des flux, que suggèrent nos résultats méritent cependant d'être confrontés à d'autres situations réelles. L'hétérogénéité des flux de pollen détectés au niveau de chaque arbre mère montre en effet qu'il existe une grande variabilité de la dispersion. Existe-t-il pour autant une variabilité aussi importante pour la structuration spatiale? Une manière d'appréhender cette variabilité serait de générer des peuplements par simulation en utilisant un modèle spatialisé, dans lequel l'un des intrants majeurs serait les flux par pollen et par graines. Ces simulations permettraient de préciser les relations entre asymétrie de flux et structure spatiale d'une part, et consanguinité et structure spatiale d'autre part.

\section{REMERCIEMENTS}

Nous remercions le Bureau des Ressources Génétiques (BRG) ainsi que l'Union Européenne (projet BIOTECH BIO04 CT96 1706) pour le soutien financier accordé à ces recherches. Nous adressons également nos plus vifs remerciements à l'Office national des forêts pour la mise à disposition de la parcelle 26 de la forêt de la Petite Charnie, à des fins expérimentales et son aide technique au cours de ce projet.

\section{RÉFÉRENCES}

Bacilieri R., Labbé T., Kremer A., Intraspecific genetic structure in a mixed population of Quercus petraea (Matt.) Liebl. and Q. robur L. Heredity 73 (1994) 130-141.

Bacilieri R., Ducousso A., Kremer A., Genetic, morphological, ecological and phenological differentiation between Quercus petraea (Matt.) Liebl. and Quercus robur L. in a mixed stand of Northwest of France, Silvae Genet. 44 (1) (1995) 1-10.

Berg E., Hamrick J.L., Fine-scale genetic structure of a turkey oak forest, Evolution 49 (1995) 110-120.

Bodénès C., Laigret F., Kremer A., Inheritance and molecular variations of PCRSSCP fragments in pedunculate oak (Quercus robur L.), Theor. Appl. Genet. 93 (1996) 348-354.

Campbell D.R., Comparing pollen dispersal and gene flow in a natural population, Evolution 45 (1991) 1965-1968.

Cliff A.D., Ord J.K., Spatial processes. Models and applications. Pion, Londres, $2^{\mathrm{e}}$ éd, (1981). 
Dow B.D., Ashley M.V., Howe H.F., Characterisation of highly variable (GA/CT)n microsatellites in the bur oak, Quercus macrocarpa, Theor. Appl. Genet. 91 (1995) 137-141.

Dow B.D., Ashley M.V., Microsatellite analysis of seed dispersal and parentage of saplings in bur oak Quercus macrocarpa, Mol. Ecol. 5 (1996) 615-627.

Doyle J.J., Doyle J.L., A rapid DNA isolation procedure for small quantities of fresh leaf tissue, Phytochem. Bull. 19 (1987) 11-15.

Dumolin-Lapègue S., Demesure B., Fineschi S., Le Corre V., Petit R.J., Phylogeographic structure of white oaks throughout the European continent, Genetics 146 (1997) 1475-1487.

Durel C.E., Bertin P., Kremer A., Relationship between inbreeding depression and inbreeding coefficient in maritime pine (Pinus pinaster), Theor. Appl. Genet. 92 (1996) 347-356.

El Mousadik A., Petit R.J., Chloroplast DNA phylogeography of the argan tree of Morocco, Molecular Ecology 5 (1996) 547-555.

Ennos R. A., Estimating the relative rates of pollen and seed migration among plant populations, Heredity 72 (1994) 250-259.

Goudet J., Raymond M., De Meeus T., Rousset F., Testing differentiation in diploid populations, Genetics 144 (1996) 1933-1940.

Kremer A., Petit R.J., Zanetto A., Fougère V., Ducousso A., Wagner D., Chauvin C., Nuclear and organelle gene diversity in Quercus petraea and Quercus robur, in : Müller-Starck G., Ziehe M.(Eds), Genetic variation in european populations of forest trees, Sauerländer's Verlag, Frankfurt am Main, 1991, pp. 141-166.

Lanier L., Précis de sylviculture, Editions de l'Engref, Nancy, 1994.

Le Corre V., Dumolin-Lapègue S., Kremer A., Genetic variation at allozyme and RAPD loci in sessile oak Quercus petraea (Matt.) Liebl. : the role of history and geography. Mol. Ecol. 6 (1997) 519-529.

Leonardi S., Menozzi P., Spatial structure of genetic variability in natural stands of Fagus sylvatica L. (beech) in Italy, Heredity 77 (1996) 359-368.

Merzeau D., Comps B., Thiébaut B., Cuguen J., Letouzey J., Genetic structure of natural stands of Fagus sylvatica L. (beech) Heredity 72 (1994) 269-277.

Petit R.J., Kremer A., Wagner D.B., Geographic structure of chloroplast DNA polymorphism in European oaks, Theor. Appl. Genet. 87 (1993) 122-128.

Schnabel A., Laushman R.H., Hamrick J.L., Comparative genetic structure of two co-occuring tree species, Maclura pomifera (Moraceae) and Gleditsia triachantos (leguminosae), Heredity 67 (1991) 357-364.

Slatkin M., A measure of population subdivision based on microsatellite allele frequencies, Genetics 139 (1995) 457-462.

Sokal R.R., Oden N.L., Spatial autocorrelation in biology. 1. Methodology, Biol. J. Linnean Soc. 10 (1978) 199-228.

Steinkellner H., Fluch S., Turetschek E., Lexer C., Streiff R., Kremer A., Burg K., Gloessl J., Identification and characterization of (GA/CT)n microsatellite loci from Quercus petraea, Plant Mol. Biol., 33 (1997) 1093-1096.

Streiff R., San Cristobal M., Kremer A., Paternity assignment, paternal identity and male mating success in a mixed stand of Quercus robur L. and Quercus petraea (Matt.) Liebl., Genetics (1998a) (submitted).

Streiff R., Labbé T., Bacilieri R., Steinkellner H., Gloessl J., Kremer A., Within population genetic structure in Quercus robur L. and Quercus petraea (Matt.) Liebl. assessed with isozymes and microsatellites, Mol. Ecol. 7 (1998b) 317-328.

Valdes A.M., Slatkin M., Freimer N.B., Allele frequencies at microsatellite loci : the stepwise mutation model revisited, Genetics 133 (1993) 737-749.

Weir B.S., Cockerham C.C., Estimating $F$-statistics for the analysis of population structure, Evolution 38 (1984) 1358-1370 
Wright S., Evolution and the Genetics of populations. Vol 2. The theory of gene frequencies, The University of Chicago Press, Chicago, 1969

Zanetto A., Roussel G., Kremer A., Geographic variation of inter-specific differentiation between Quercus robur L. and Quercus petraea (Matt.) Liebl., Forest Genetics 1 (1994) 111-123

Zanetto A., Kremer A., Geographic structure of gene diversity in Quercus petraea (Matt.) Liebl. I. Monolocus patterns of variation, Heredity 75 (1995) 506-517 